\title{
Objective assessment based on motion-related metrics and technical performance in laparoscopic suturing
}

\author{
Juan A. Sánchez-Margallo • Francisco M. Sánchez-Margallo • \\ Ignacio Oropesa · Silvia Enciso · Enrique J. Gómez
}

\begin{abstract}
Purpose The aim of this study is to present the construct and concurrent validity of a motion-tracking method of laparoscopic instruments based on an optical pose tracker and determine its feasibility as an objective assessment tool of psychomotor skills during laparoscopic suturing.

Methods A group of novice (<10 laparoscopic procedures), intermediate (11-100 laparoscopic procedures) and experienced (>100 laparoscopic procedures) surgeons performed three intracorporeal sutures on an ex vivo porcine stomach. Motion analysis metrics were recorded using the proposed tracking method, which employs an optical pose tracker to determine the laparoscopic instruments' position. Construct validation was measured for all 10 metrics across the three groups and between pairs of groups. Concurrent validation was measured against a previously validated suturing checklist. Checklists were completed by two independent surgeons over blinded video recordings of the task.

Results Eighteen novices, 15 intermediates and 11 experienced surgeons took part in this study. Execution time and path length travelled by the laparoscopic dissector presented construct validity. Experienced surgeons required signifi-
\end{abstract}

cantly less time $(p<0.008)$, travelled less distance using both laparoscopic instruments $(p<0.013)$ and made more efficient use of the work space $(p<0.018)$ compared with novice and intermediate surgeons. Concurrent validation showed strong correlation between both the execution time and path length and the checklist score $(r=-0.712$ and $r=-0.731, p<0.001$ ).

Conclusions The suturing performance was successfully assessed by the motion analysis method. Construct and concurrent validity of the motion-based assessment method has been demonstrated for the execution time and path length metrics. This study demonstrates the efficacy of the presented method for objective evaluation of psychomotor skills in laparoscopic suturing. However, this method does not take into account the quality of the suture. Thus, future works will focus on developing new methods combining motion analysis and qualitative outcome evaluation to provide a complete performance assessment to trainees.

Keywords Laparoscopic surgery - Surgical training . Objective assessment - Motion metrics - Intracorporeal suturing $\cdot$ Suturing checklist

\section{Introduction}

Laparoscopic surgery is a high demanding surgical approach concerning technical requirements for surgeons [1]. This presents some challenges for surgeons caused by insufficient ergonomic criteria, reduced degrees of freedom and decreased tactile feedback, which may provide suboptimal operating conditions. These aspects require new surgical skills for the surgeon, who must be trained and assessed in order to become proficient, enabling him/her to perform safe surgical interventions. Traditional subjective assessment 
methods of trainees are no longer adequate for surgical training [2,3]. Besides, reduced working hours [4] and demands from surgeons and medical associations $[5,6]$ mean that more objective assessment tools able to accredit surgeons as technically competent are required.

Evaluation of surgeons' technical skills during their training program is an essential part of a comprehensive assessment of their surgical proficiency. Surgical simulators are effective tools for training and assessment, mainly during the early phases of the training curricula. They offer a pressurefree environment and avoid putting patients at risk. They have the potential to provide a reproducible setup for all trainees to objectively assess surgical performance. Studies have shown that skills acquired during simulation training could be transferred to the operating room [7]. A number of objective assessment tools for surgical skills, including motion analysis, have been developed for use with surgical simulators [8,9]. Most of these systems are based on scoring methods [10], carousel of tasks with sensors for monitoring metrics [11] and instrument motion tracking [12-15]. A simulator can be considered an effective educational tool only if the metrics associated with its use provide meaningful and accurate information to the trainee [16].

Evidence exists to validate motion analysis for use in laparoscopic skills assessment [17]. Indicative of its growing validity is that most available virtual reality simulators and hybrid models have incorporated motion metrics into the measurements reported to the user $[8,9]$. Motion analysis methods compute metrics to quantify motion information concerning the use of the surgical instruments to establish the surgical dexterity level of surgeons. They seek to determine aspects that indicate the difference between performances at various levels of proficiency. Despite the increasing availability of simulators that include instrument motion analysis, they usually provide traditional metrics such as path length, motion smoothness and number of movements. Therefore, new metrics should be explored and validated, which can provide additional assessment information.

Laparoscopic suturing and knot tying is a potentially difficult task that demands intensive practice to master the required skills. Quality of technical performance in laparoscopic suturing can be assessed using scoring systems such as checklists [18]. Other evaluation methods use motion analysis metrics [12,19] or computerized systems [20] for psychomotor skills assessment. However, studies that analyze the relationship of these motion analysis metrics with the assessment of quality in laparoscopic technical performance are scarce.

In previous studies, a method for motion analysis of laparoscopic instruments based on an optical pose tracker was presented and technically validated [21,22]. This approach tries to addresses certain limitations of other solutions for motion tracking of laparoscopic instruments such as ferromagnetic interferences, the need of wires or major internal modifications of the instruments. The purpose of this work is to establish the construct and concurrent validity of this motion analysis method and determine its feasibility as an objective assessment tool of psychomotor skills during laparoscopic suturing. Besides, the relationship between motion-related metrics and the technical assessment of intracorporeal suturing performance using a checklist is presented.

\section{Material and methods}

\section{Participants}

Surgeons from different training courses in urological and gynecological minimally invasive surgical techniques held at the Jesús Usón Minimally Invasive Surgery Centre (JUMISC, Cáceres, Spain) were asked to participate in this study. All participants completed a short questionnaire detailing demographic information, dominant hand and prior experience in laparoscopic surgery. Subjects were classified according to their laparoscopic experience as experienced (E, $>100$ laparoscopic procedures), intermediates (I, 10-100 laparoscopic procedures) and novices (,$<10$ laparoscopic procedures).

\section{System description}

Training tasks were performed on a laparoscopic box trainer (SimulapßB); JUMISC, Cáceres, Spain) [23,24]. Ergonomic conditions were standardized for all subjects. The height of the surgical table was adjusted according to that of the subject, and the monitor was placed at eye level for each surgeon. Entry ports for both instruments were set in identical spatial configuration and with the camera system focused on the work area. In order to record the movements of the instruments during suturing performance, a motion analysis method for laparoscopic instruments based on a third-generation optical pose tracker (MicronTracker(B) 3 Hx60; Claron Technology Inc., Toronto, Canada) was used (Fig. 2a) [21]. This tracking method works in the visible spectrum and uses custom-made artificial markers on the laparoscopic instrument handle as reference points (Fig. 2b). Technical validation of this tracking method for laparoscopic instruments has been presented in a previous study [22].

\section{Tasks}

Surgeons were asked to perform three intracorporeal sutures on a 30-mm incision on an ex vivo porcine stomach placed into a box trainer (Figs. 1, 2c). The suturing task consisted of four steps: passing the needle through the top and then 


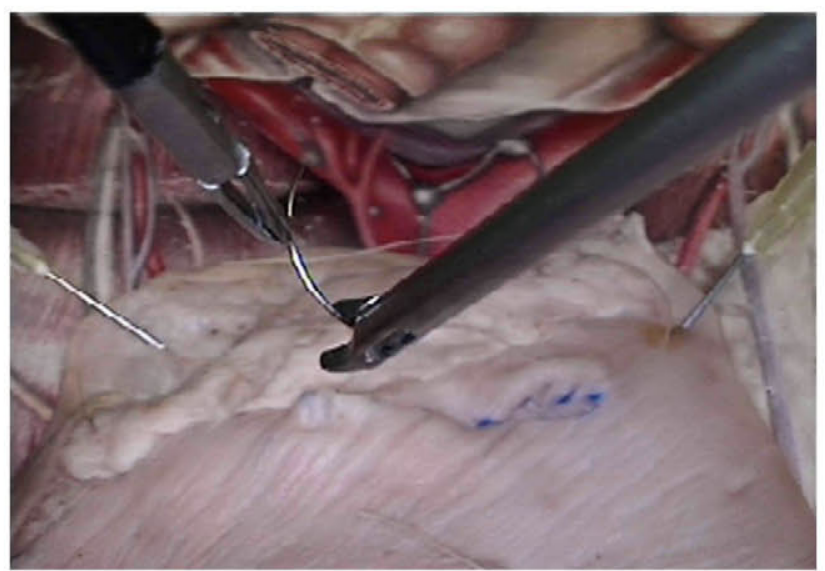

Fig. 1 Intracorporeal suturing task on an ex vivo model

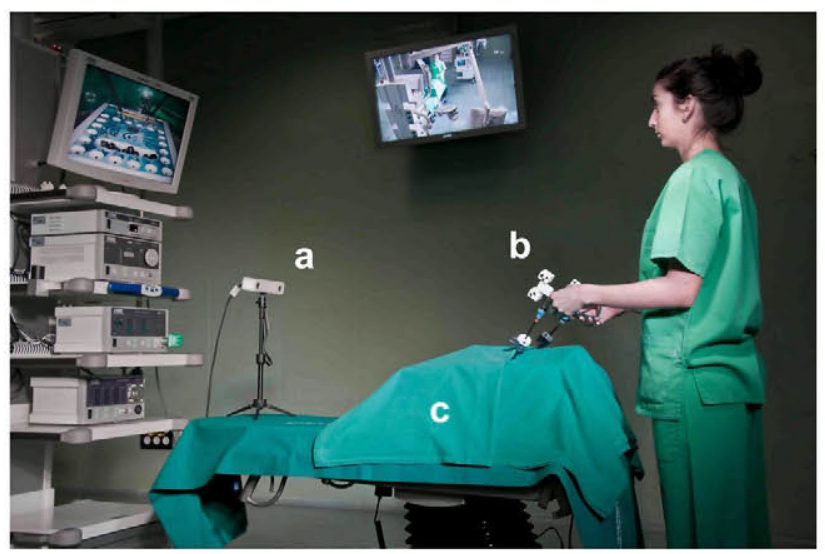

Fig. 2 Experimental setup: (a) camera system of the optical pose tracker; (b) laparoscopic instruments with artificial markers; (c) covered box trainer with the exercise displayed on the monitor

the bottom of the incision (in two steps); a double knot; and two single knots in opposite directions. For this task, subjects used a laparoscopic needle holder (Karl Storz GmbH \& Co. $\mathrm{KG}$, Tuttlingen, Germany) with the dominant hand and a laparoscopic dissector (Richard Wolf $\mathrm{GmbH}$, Knittlingen, Germany) with the non-dominant hand. Suture material (2-0 $26 \mathrm{~mm}$ Safil@, B. Braun Medical Ltd, Melsungen, Germany) was standardized for all tasks.

\section{Assessment}

Ten metrics of psychomotor skills were computed, derived from the motion of the laparoscopic instruments' tip. Some of them such as time, path length, depth, speed, acceleration and motion smoothness have been extensively used [14,15,25]. Other metrics such as idle time, economy of area, economy of volume and bimanual dexterity have been introduced recently and thus still require further validation $[14,26]$. The economy of area is the relationship between the maximum surface area occupied by the instrument in the task plane and the total path length. Similarly, economy of volume is the relationship between the maximum volume occupied by the instrument in the setting and the total path length [14]. Both metrics measure the space-usage efficiency in the box trainer. The bimanual dexterity is the surgeon's ability to control two instruments at the same time. It is found by calculating the correlation between the velocity of the instrument tip controlled by the left and the right hand [26]. The analyzed motion metrics were organized into two categories: timerelated metrics and space-related metrics. Performance of each task was videotaped and synchronized with the instruments motion recordings.

In addition, the performance of the suturing task was rated using a previously validated suturing checklist [18,27]. This checklist scores technical features of the task such as needle position and driving, pulling through of the suture and the technique and quality of the intracorporeal knots. This study does not include slip knots, and therefore items 25 (knot left loose to slip) and 26 (knot slippage attempts 3 or $<3$ ) of the checklist have not been considered, resulting in a maximum final score of 27 . Two experienced surgeons scored the recorded tasks of each subject. Both surgeons were blinded to their respective findings, as well as to the identity and experience level of each subject.

\section{Statistical analysis}

For statistical analysis, nonparametric tests of significance were used. All statistical analyses were carried out using the software SPSS version 19.0 (SPSS Inc., Chicago, IL). Construct validation was measured for each motion analysis metric. Significant differences in performance were sought along the three groups by Kruskal-Wallis analysis. If the groups differed significantly, the Mann-Whitney $U$ test was used for pairwise comparison to test the difference between each study group.

Concurrent validation was established between metrics calculated by the system and the scores given by the surgeons using the checklists. Cronbach's alpha test was used to measure the extent of agreement between the two raters (inter-examiner reliability). A value of alpha equal to 0.8 was accepted as a threshold for good reliability [2]. Correlation between motion-based assessment metrics and the mean score of each suturing technical feature was analyzed using Spearman's rank correlation coefficient (rho, $\rho$ ). Correlation values between 0.4 and 0.7 were considered as moderately good, whilst values $>0.7$ were considered to indicate a strong correlation between the two modalities of assessment [28]. For all tests, $p<0.05$ was considered statistically significant. 


\section{Results}

Forty-four surgeons took part in the study: 11 experienced surgeons, 15 intermediates and 18 novices. The average age of the participants was 35.2 years $(S D=9.37)$ and the male/female ratio 29/15. Two participants were left-handed and therefore performed the suturing task holding the laparoscopic needle holder on their left hand. All participants were able to complete the tasks, without imposing time restrictions.

\section{Construct validation}

All time-related assessment metrics except bimanual dexterity presented statistically significant differences between the group of novice and intermediate surgeons for the use of both instruments $(p<0.031)$ and between the group of novice and experienced surgeons for the needle holder ( $p<0.007$; Fig. 3). Execution time showed significant differences between the three groups of surgeons $(p<0.008)$.

With respect to space-related metrics, all metrics except depth showed significant differences between novices and experienced surgeons $(p<0.008)$, as well as between intermediates and experienced surgeons for the use of both instruments ( $p<0.049$; Fig.4). Experienced surgeons travelled significantly less distance using both laparoscopic instruments and made more efficient use of the work space. Path length travelled by the laparoscopic dissector presented statistically significant differences between the three groups of surgeons $(p<0.023)$.

\section{Concurrent validation}

The inter-examiner reliability was 0.974 for the evaluation using the suturing checklist. Both experienced and intermediate surgeons scored significantly higher than novice surgeons $(\mathrm{N}: 16.50 \pm 1.60, \mathrm{I}: 21.55 \pm 1.87, \mathrm{E}: 23.25 \pm 2.20$; $p<0.001)$. However, there were no statistically significant differences between the experienced and intermediate groups.

All technical features rated by the suturing checklist were related to time and path length showing a strong correlation with needle position (Tables 1, 2). Needle positioning and driving were also related to economy of area and economy of volume. The pulling maneuver correlated with all timerelated metrics, except bimanual dexterity.

\section{Discussion}

The presented motion analysis method for laparoscopic instruments has been demonstrated to be a valid tool for objective assessment of psychomotor skills in laparoscopic suturing. Results showed that execution time and path length travelled by the laparoscopic dissector are good predictors for the experience level of a surgeon. These metrics showed a good correlation between them and the technical parameters evaluated by the suturing checklist, making them potential useful indicators of the quality of the technical performance in laparoscopic suturing. Moreover, unlike motion analysis, the suturing checklist was not able to distinguish between intermediate and expert surgeons. Therefore, motion analysis of surgical instruments could be considered as a valuable complementary objective assessment tool to the suturing checklist for laparoscopic suturing tasks.

The time taken to perform a surgical task has usually been one of the most widely validated assessment metrics [17]. In fact, the speed in performing the task has been used traditionally as an objective measure of surgical dexterity, considering that an expert surgeon should perform a task more swiftly. Results of this study support these findings for the laparoscopic suturing task. Construct validity of execution time in intracorporeal suturing has also been reported in other studies $[12,19,25,27]$.

A similar correlation study in laparoscopic suturing was presented by Moorthy et al. [27] using an electromagnetic tracker attached to the surgeon's hands. They obtained significant differences for execution time and total path length travelled by both instruments. Our results also showed construct validity for these metrics, but only for the path length travelled by the laparoscopic dissector, similarly to what was reported by Yamaguchi et al. [19]. However, the latter study only included two experience groups, novice and experienced surgeons. They used an electromagnetic tracking system with a sensor attached to the tip of each laparoscopic instrument. Results showed that dexterity using the laparoscopic dissector rather than the needle holder is a good indicator of the level of expertise in intracorporeal suturing. This seems to indicate that surgical experience can affect surgical dexterity of each hand differently in laparoscopic suturing.

Within the group of time-related metrics, bimanual dexterity had no significant differences between any of the experience groups. However, other studies found significant differences during the performance of hand coordination and suturing exercises $[12,26]$. A possible reason of this may reside in the post-filtering stage to the data, which could have slightly reduced the motion information about each instrument and thus the correlation between them. Apart from bimanual dexterity, no statistically differences were found for the depth metric between any of the groups of surgeons. Since a defined workspace inside the simulator was established for all tasks, depth of movements for the instruments tip may not vary significantly.

Intermediate surgeons used both laparoscopic instruments with higher speed, higher acceleration and jerkier movements than the other two groups, but with no significant 

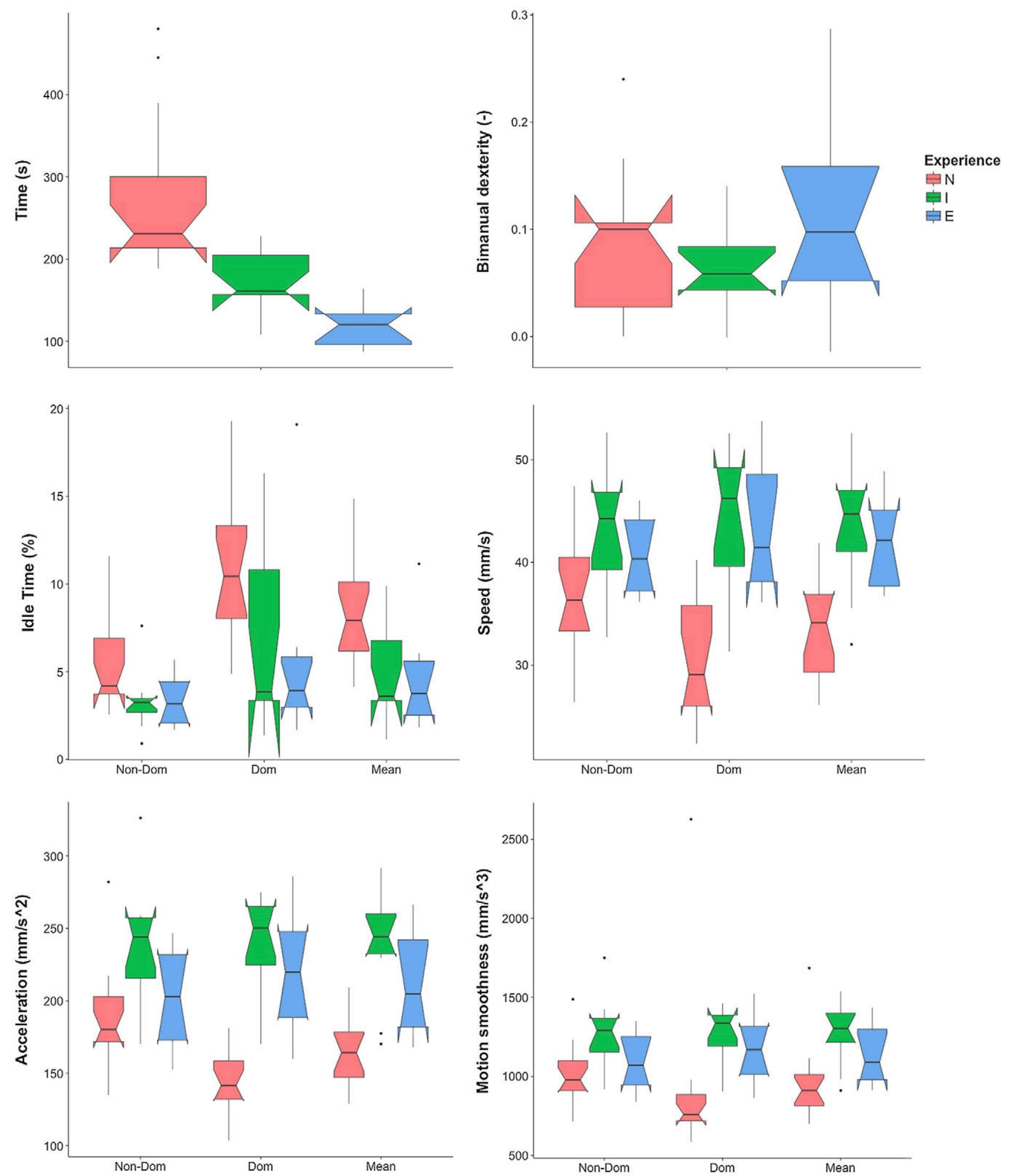

Fig. 3 Construct validation results for time-related metrics: scores for time, bimanual dexterity, idle time, speed, acceleration and motion smoothness. Results are presented as notched box plots, in which every notched box has a line marking the lower quartile, median and upper

quartile value. Whiskers represent the extent of the remaining data, with dots showing the outliers. The boxes whose notches do not overlap are significantly different $(p<0.05)$. Dom dominant hand, Non-Dom nondominant hand 

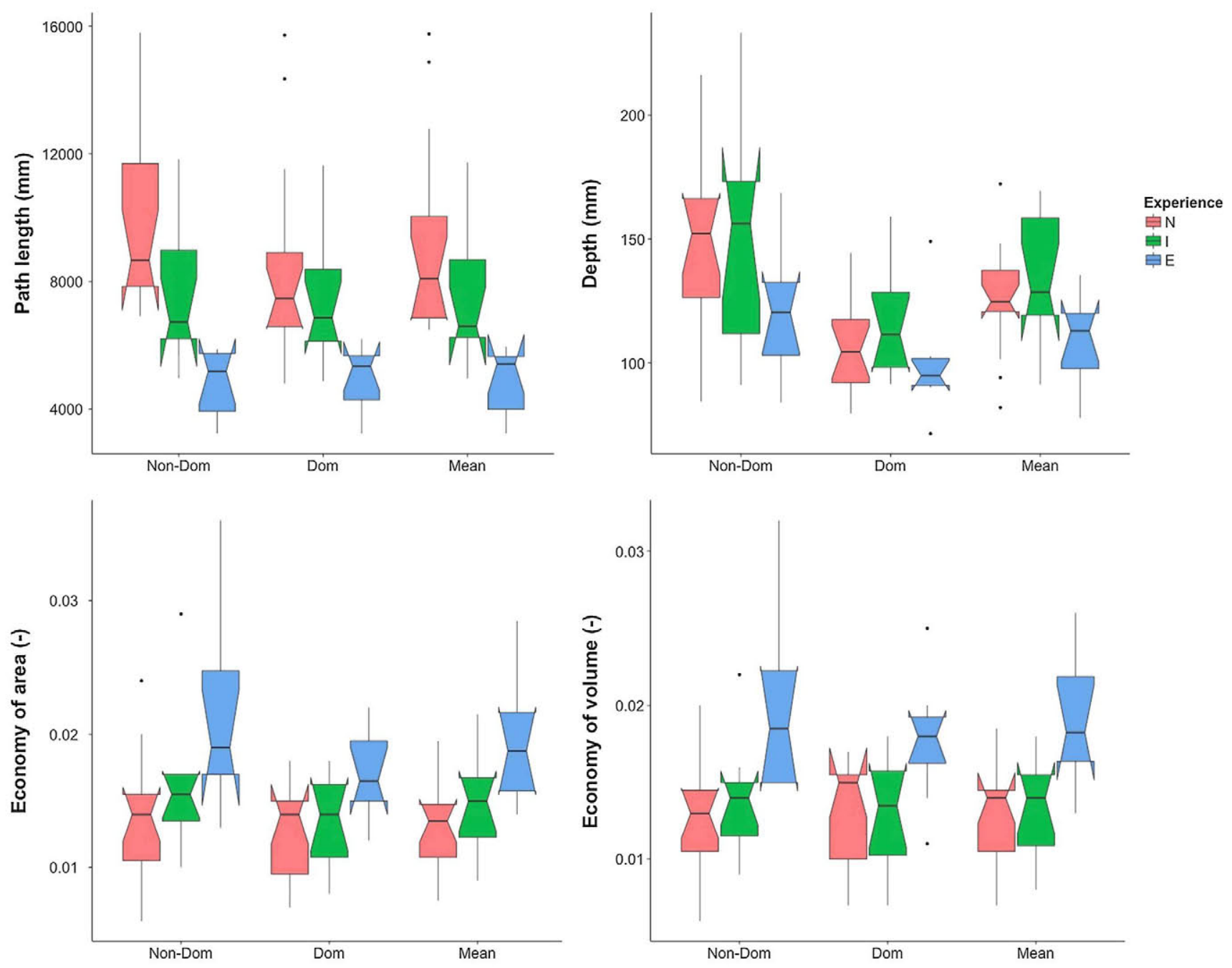

Fig. 4 Construct validation results for the space-related metrics: scores for path length, depth, economy of area and economy of volume. Results are presented as notched box plots, in which every notched box has a line marking the lower quartile, median and upper quartile value. Whiskers

represent the extent of the remaining data, with dots showing the outliers. The boxes whose notches do not overlap are significantly different $(p<0.05)$. Dom dominant hand, non-dom non-dominant hand

Table 1 Correlations between technical features rated by the suturing checklist and time-related motion metrics

\begin{tabular}{llllllr}
\hline & Time & Idle time & Speed & Acceleration & Motion smoothness & Bimanual dexterity \\
\hline Needle position & $-\mathbf{0 . 7 0 5 * *}$ & -0.295 & 0.290 & 0.349 & 0.314 & -0.029 \\
Needle driving & $-\mathbf{0 . 6 6 1 * *}$ & -0.351 & 0.295 & 0.279 & 0.196 & 0.020 \\
Pulling & $-\mathbf{0 . 4 9 1 *}$ & $-\mathbf{0 . 4 2 9 *}$ & $\mathbf{0 . 4 1 2} *$ & $\mathbf{0 . 5 2 2 *}$ & $\mathbf{0 . 6 0 0 * *}$ & -0.007 \\
Technique & $-\mathbf{0 . 5 3 6 * *}$ & -0.191 & 0.302 & 0.352 & 0.279 & -0.091 \\
Quality & $-\mathbf{0 . 4 5 7 *}$ & -0.290 & 0.142 & 0.194 & 0.086 & 0.141 \\
Total & $-\mathbf{0 . 7 1 2 * *}$ & -0.324 & 0.309 & 0.397 & 0.361 & -0.033 \\
\hline
\end{tabular}

Correlation values are presented as Spearman's rho $(r)$ and its significance $(p)$

Statistically significant values are marked in bold face. ${ }^{*} p<0.05 ; * * p<0.001$

differences with regard to the experienced group of surgeons. However, the group of intermediate surgeons travelled significantly more distance with both instruments and obtained less economy of covered area and volume than the group of expe- rienced surgeons, which leads to less efficient movements. It could be expected that more experienced surgeons would make smoother movements than less experienced ones, but results showed that novice surgeons performed the intracor- 
Table 2 Correlations between technical features rated by the suturing checklist and space-related motion metrics

\begin{tabular}{|c|c|c|c|c|}
\hline & Path length & Depth & Economy of area & Economy of volume \\
\hline Needle position & $-0.740 * *$ & -0.206 & $0.523 *$ & $0.452 *$ \\
\hline Needle driving & $-0.677 * *$ & -0.242 & $0.473^{*}$ & $0.419 *$ \\
\hline Pulling & $-0.424 *$ & -0.155 & 0.285 & 0.127 \\
\hline Technique & $-0.508 *$ & -0.211 & 0.374 & 0.371 \\
\hline Quality & $-0.506^{*}$ & -0.112 & 0.369 & 0.229 \\
\hline Total & $-0.731 * *$ & -0.228 & $0.532 *$ & $0.429 *$ \\
\hline
\end{tabular}

Correlation values are presented as Spearman's rho (r) and its significance $(p)$

Statistically significant values are marked in bold face. $* p<0.05 ; * * p<0.001$ poreal suture with the lowest rate of jerky movements, as reported by [26]. We hypothesize that lower experience of the novice group may make them execute the maneuvers in a more careful manner.

Regarding the assessment of suturing performance by means of the suturing checklist, as in the study of Kroeze et al. [18], there were no statistically significant differences between intermediate and expert surgeons, in contrast to [27] who obtained significant difference across the three analyzed groups. These conflicting results may be because the suturing checklist only takes into account technical features, overlooking other parameters such as efficiency or depth perception, which are evaluated by other assessment methods such as the Global Operative Assessment of Laparoscopic Skills (GOALS) scoring system [29]. Moreover, both groups of intermediate and expert surgeons had a prior experience in laparoscopic suturing and therefore were familiar with the technical steps of this task.

Concurrent validity of the motion-based assessment method has been demonstrated for the execution time and path length metrics. A strong correlation between the checklist score and the distance travelled by both laparoscopic instruments has been also reported by [27], but not for the execution time. This may be due to the fact that in said study, needle positioning was not considered for assessment. Results showed that better technique regarding the placement of the needle and total score for the suturing checklist led to a lower execution time and path travelled by the laparoscopic instruments during laparoscopic suturing. Both an adequate positioning of the needle and an accurate driving of the needle involved fewer and more focused movements of the instruments, and therefore a better economy of the area and volume occupied by both instruments. In addition, a proper execution of the technique to pull the suture through the incision was related to a decrease in the total execution time and idle times during the task performance, but also to an increase in speed and acceleration of both instruments as well as jerky movements.

One of the main challenges in the presented method of laparoscopic technical skills assessment based on instrument motion analysis is transferring it to an actual clinical set- ting. This would involve analyzing the most suitable position of the tracking camera system inside the operating room in order to avoid occlusions in a potential crowded surgical environment. Additionally, easily sterilizable, tough and lightweight material for the artificial markers placed on the surgical instruments should be sought. On the other hand, the presented motion-based assessment method provides a quantitative measure of technical skills, but no information on the outcome of the completed task. The quality of the outcome is a fundamental part for a comprehensive evaluation of the intracorporeal suturing performance, and we consider this as the next step in the evaluation process and as a complement to the evaluation of psychomotor skills and technical performance presented in this study.

In conclusion, this study has demonstrated the feasibility of using the presented motion analysis method for assessing psychomotor skills of surgeons during laparoscopic suturing. Results have established the validity of execution time and path length travelled by laparoscopic instruments as objective assessment metrics for this task.

Acknowledgements This work has been partially funded by the Autonomous Community of Extremadura, Spain, and the European Social Fund (PO14034).

\section{Compliance with ethical standards}

Conflict of interest J. A. Sánchez-Margallo, F. M. Sánchez-Margallo, I. Oropesa, S. Enciso and E. J. Gómez declare that they have no conflict of interest.

Ethical approval This article does not contain any studies with human participants or animals performed by any of the authors.

Informed consent Informed consent was obtained from all individual participants included in the study.

\section{References}

1. Smith CD, Farrell TM, McNatt SS, Metreveli RE (2001) Assessing laparoscopic manipulative skills. Am J Surg 181(6):547-550

2. van Hove PD, Tuijthof GJM, Verdaasdonk EGG, Stassen LPS, Dankelman J (2010) Objective assessment of technical surgical skills. Br J Surg 97(7):972-987 
3. Fried GM, Feldman LS (2008) Objective assessment of technical performance. World J Surg 32(2):156-160

4. Parsons BA, Blencowe NS, Hollowood AD, Grant JR (2011) Surgical training: the impact of changes in curriculum and experience. J Surg Educ 68(1):44-51

5. Roberts KE, Bell RL, Duffy AJ (2006) Evolution of surgical skills training. World J World J Gastroenterol 12(20):3219-3224

6. Ritchie WP (2004) Basic certification in surgery by the American Board of Surgery (ABS). What does it mean? Does it have value? Is it relevant? A personal opinion. Ann Surg 239(2):133-139

7. Kurashima Y, Feldman LS, Kaneva PA, Fried GM, Bergman S, Demyttenaere SV, Li C, Vassiliou MC (2014) Simulation-based training improves the operative performance of totally extraperitoneal (TEP) laparoscopic inguinal hernia repair: a prospective randomized controlled trial. Surg Endosc 28(3):783-788

8. Sánchez-Margallo JA, Sánchez-Margallo FM, Oropesa I, Gómez EJ (2014) Systems and technologies for objective evaluation of technical skills in laparoscopic surgery. Minim Invasive Ther Allied Technol 23(1):40-51

9. Oropesa I, Sánchez-González P, Lamata P, Chmarra MK, Pagador JB, Sánchez-Margallo JA, Sánchez-Margallo FM, Gómez EJ (2011) Methods and tools for objective assessment of psychomotor skills in laparoscopic surgery. J Surg Res 175(1):e81-e95

10. Vassiliou MC, Feldman LS, Fraser SA, Charlebois P, Chaudhury P, Stanbridge DD, Fried GM (2007) Evaluating intraoperative laparoscopic skill: direct observation versus blinded videotaped performances. Surg Innov 14(3):211-216

11. Hasson HM (2008) Simulation training in laparoscopy using a computerized physical reality simulator. JSLS 12(4):363-367

12. Pérez F, Ordorica RM, Oropesa I, Zalles CR, Minor A (2015) Face, content, and construct validity of the EndoViS training system for objective assessment of psychomotor skills of laparoscopic surgeons. Surg Endosc 29(11):3392-3403

13. Sánchez-Margallo JA, Sánchez-Margallo FM, Pagador JB, Gómez EJ, Sánchez-González P, Usón J, Moreno J (2011) Video-based assistance system for training in minimally invasive surgery. Minim Invasive Ther Allied Technol 20(4):197-205

14. Oropesa I, Sánchez-González P, Chmarra MK, Lamata P, Fernández A, Sánchez-Margallo JA, Jansen FW, Dankelman J, SánchezMargallo FM, Gómez EJ (2013) EVA: laparoscopic instrument tracking based on endoscopic video analysis for psychomotor skills assessment. Surg Endosc 27(3):1029-1039

15. Chmarra MK, Klein S, de Winter JCF, Jansen F-W, Dankelman J (2010) Objective classification of residents based on their psychomotor laparoscopic skills. Surg Endosc 24(5):1031-1039

16. Fried GM, Feldman LS, Vassiliou MC, Fraser SA, Stanbridge D, Ghitulescu G, Andrew CG (2004) Proving the value of simulation in laparoscopic surgery. Ann Surg 240(3):518-528

17. Mason JD, Ansell J, Warren N, Torkington J (2013) Is motion analysis a valid tool for assessing laparoscopic skill? Surg Endosc 27(5):1468-1477
18. Kroeze SGC, Mayer EK, Chopra S, Aggarwal R, Darzi A, Patel A (2009) Assessment of laparoscopic suturing skills of urology residents: a Pan-European study. Eur Urol 56(5):865-872

19. Yamaguchi S, Yoshida D, Kenmotsu H, Yasunaga T, Konishi K, Ieiri S, Nakashima H, Tanoue K, Hashizume M (2011) Objective assessment of laparoscopic suturing skills using a motion-tracking system. Surg Endosc 25(3):771-775

20. Uemura M, Yamashita M, Tomikawa M, Obata S, Souzaki R, Ieiri S, Ohuchida K, Matsuoka N, Katayama T, Hashizume M (2015) Objective assessment of the suture ligature method for the laparoscopic intestinal anastomosis model using a new computerized system. Surg Endosc 29(2):444-452

21. Sánchez-Margallo JA, Sánchez-Margallo FM, Pagador Carrasco JB, Oropesa García I, Gómez Aguilera EJ, Moreno Del Pozo J (2013) Usefulness of an optical tracking system in laparoscopic surgery for motor skills assessment. Cir Esp 92(6):421-428

22. Sánchez-Margallo JA, Sánchez-Margallo FM, Pagador JB, Oropesa I, Lucas M, Gómez EJ, Moreno J (2013) Technical evaluation of a third generation optical pose tracker for motion analysis and image-guided surgery. Lect Notes Comput Sci 7761:75-82

23. Enciso Sanz S, Sánchez Margallo FM, Díaz-Güemes MartínPortugués I, Usón Gargallo J (2012) Preliminary validation of the Simulap ${ }^{\circledR}$ physical simulator and its assessment system for laparoscopic surgery. Cir Esp 90(1):38-44

24. Sánchez-Margallo FM, Díaz-Güemes I, Pérez FJ, Sánchez MA, Loscertales B, Usón J (2009) Preliminary results with a training program for thoracoscopic atrial fibrillation therapy. Surg Endosc 23(8):1882-1886

25. Pagador JB, Sánchez-Margallo FM, Sánchez-Peralta LF, SánchezMargallo JA, Moyano-Cuevas JL, Enciso-Sanz S, Usón-Gargallo J, Moreno J (2012) Decomposition and analysis of laparoscopic suturing task using tool-motion analysis (TMA): improving the objective assessment. Int J Comput Assist Radiol Surg 7(2):305313

26. Hofstad EF, Våpenstad C, Chmarra MK, Langø T, Kuhry E, Mårvik R (2013) A study of psychomotor skills in minimally invasive surgery: what differentiates expert and nonexpert performance. Surg Endosc 27(3):854-863

27. Moorthy K, Munz Y, Dosis A, Bello F, Chang A, Darzi A (2004) Bimodal assessment of laparoscopic suturing skills: construct and concurrent validity. Surg Endosc 18(11):1608-1612

28. Aggarwal R, Grantcharov T, Moorthy K, Milland T, Papasavas P, Dosis A, Bello F, Darzi A (2007) An evaluation of the feasibility, validity, and reliability of laparoscopic skills assessment in the operating room. Ann Surg 245(6):992-999

29. Vassiliou MC, Feldman LS, Andrew CG, Bergman S, Leffondré K, Stanbridge D, Fried GM (2005) A global assessment tool for evaluation of intraoperative laparoscopic skills. Am J Surg 190(1):107-113 